\title{
Content-Based Image Indexing and Retrieval: A Syntactical Approach
}

\author{
S. HE and N. ABE \\ Kyushu Institute of Technology \\ Kawazu 680-4, Iizuka 820-8502, Japan \\ E-mail: hesj@cse.kyutech.ac.jp
}

\begin{abstract}
This paper presents a syntactical approach to the content-based indexing and retrieval of images. Studies on the content-based indexing/retrieval of images were motivated to derive from the visual features inside images a set of objective criteria for the indexing/retrieval of the images. The existing content-based image indexing and retrieval methods, however, use in an unstructured manner the visual features such as edge elements, color histogram, texture distribution, and so on. As a result, both indexing and retrieval are not efficient and effective, compared with the text-based methods. A new hierarchical structure representation is presented in this paper. Since the hierarchical structure is generated with a data-driven approach, an objective description on an image can be obtained. The potential application of the hierarchical structure to the content-based image indexing and retrieval is discussed.
\end{abstract}

\section{Introduction}

The traditional hierarchical structure representation has been defined on the basis of human perception [1]. It might be used as a model for a topdown approach to the image analysis but can never be applied to solving the content-based indexing and retrieval problems. This is because the content-based indexing and retrieval of an image basically require an objective description on the image. The traditional hierarchical structure representation, however, is strongly dependent on the subjective interpretation given by each individual person. Given a single image, a different person may give a different interpretation to the image and thus may represent the image with a completely different hierarchical structure.

Studies on the content-based indexing of images are originally motivated by the needs of eliminating the subjective biases involved in the text-based indices. Most of the existing content-based indexing approaches, however, are limited to the use of low-level visual features [2]. Since the low-level visual features are usually used in an unstructured and not well-organized manner, the retrieval is no more than an inefficient pattern matching process. 
This paper presents a new hierarchical structure representation. The new hierarchical structure representation is generated with a completely data-driven approach and hence is an objective description on an image. The rest of this paper is organized as follows. Section 2 describes in detail the process of how the new hierarchical structure representation is defined. Section 3 shows the algorithms for the generation of our new hierarchical structure representation. Section 4 discusses with experimental results on the potential application of our hierarchical structure representation to the content-based indexing and retrieval. Section 5 concludes the paper with the discussion on the future work.

\section{Hierarchical Structure Representation}

In this section, we first give a formal definition on the primitive components of our structure representation and then define two basic spatial rerationships. Finally, we define the new structure representation by generalizing the definition of the basic spatial relationships.

\subsection{Generalized connected component (GCC)}

\subsubsection{Definition of $G C C$}

Definition 1: A GCC $\sigma$ is a set of pixels so that

$$
\forall p \exists q(P(p, q) \wedge M(q, \sigma) \wedge C(p, q, f) \Rightarrow M(p, \sigma))
$$

where $p$ and $q$ are two different pixels, $P(p, q)$ means that $p$ and $q$ share a kind of uniform property such as intensity, color, etc., $M(q, \sigma)$ means $q \in \sigma$, and $C(p, q, f)$ is a connectivity constraint between $p$ and $q$.

The connectivity constraint is recursively defined with the following boundary condition:

$$
\forall u \forall v(N(u, v, f) \wedge P(u, v) \Rightarrow C(u, v, f))
$$

where $N(u, v, f)$ means that pixels $u$ and $v$ neighbor with each other, $f$ specifies the neighborship ${ }^{4}$. The recursion part is:

$$
\forall u \forall v \exists r(P(u, r) \wedge P(r, v) \wedge C(u, r, f) \wedge C(r, v, f) \Rightarrow C(u, v, f))
$$

\subsubsection{Region/Segment vs, GCC}

According to Def. 1, a GCC is a pixel group. Since region and segment have been widely-used concepts for pixel groups, it is naturally enquired why it is necessary to introduce a new concept. The reason that GCC's are not replaceable by regions/segments is as follows: 
- semantics levels: region is a high-level concept, while segment is a low-level concept.

- connectivity: region completely satisfies the connectivity constraints in Def. 1, while segment partially satisfies the constraints.

GCC is a concept in the middle position in terms of both the semantics levels and pixels connectivity.

\subsection{Basic Spatial Relationships}

Given an image $\Delta$, let $\Delta^{\prime}$ denote the border of $\Delta . \Sigma=\left\{\sigma_{i}|1 \leq i \leq| \Sigma \mid\right\}$ is obtained from $\Delta$.

Definition 2: The border $\sigma_{i}^{\prime}$ of $\sigma_{i}$ is a subset of $\sigma_{i}$ such that $\sigma_{i}^{\prime}=\{p \mid$ $\left.\exists q \in \Delta^{\prime} \exists \pi \sigma_{i} \cap \pi=\{p\}\right\}$, where $\pi$ is a path ${ }^{5}$ between $p$ and $q$ and $\{p\}$ denotes a set with a single pixel $p$.

\section{Definition 3:}

$$
\operatorname{surrounded}\left(\sigma_{i}, \sigma_{j}\right) \Leftrightarrow \forall p\left(N_{b}\left(p, \sigma_{i}^{\prime}\right) \Rightarrow M\left(p, \sigma_{j}\right)\right)
$$

where surrounded $\left(\sigma_{i}, \sigma_{j}\right)$ means that $\sigma_{i}$ is surrounded by $\sigma_{j}$ and $\sigma_{j}$ surrounds $\sigma_{i}$. Note that (4) implies $\sigma_{i}^{\prime} \cap \Delta^{\prime}=\phi$.

Definition 4:

$$
\begin{array}{r}
j \operatorname{oint}\left(\sigma_{i}, \sigma_{j}\right) \Leftrightarrow \quad\left(\exists p\left(N_{b}\left(p, c_{i}^{\prime}\right) \wedge M\left(p, \sigma_{j}\right)\right) \vee\right. \\
\left.\exists q\left(N_{b}\left(q, \sigma_{j}^{\prime}\right) \wedge M\left(q, \sigma_{i}\right)\right)\right)
\end{array}
$$

where joint $\left(\sigma_{i}, \sigma_{j}\right)$ means that $\sigma_{i}$ and $\sigma_{j}$ are joint with each other.

The meaning of $N_{b}$ in (4) and (5) is as follows. Given a pixel $q \in \sigma_{i}^{\prime}$, let $q_{1}, q_{2}, \cdots, q_{8}$ denote the eight neighbors of $q$ in clockwise order. Suppose $\sigma_{i}^{\prime}$ is tracked clockwise. $N_{b}\left(q_{m}, \sigma_{i}^{\prime}\right)$ is true iff $q_{m} \notin \sigma_{i}^{\prime}$ but $q_{(m+1)(\bmod 8)} \in$ $\sigma_{i}^{\prime}$. Obviously, joint $\left(\sigma_{i}, \sigma_{j}\right)=j$ joint $\left(\sigma_{j}, \sigma_{i}\right)$ but $\operatorname{surrounded}\left(\sigma_{i}, \sigma_{j}\right) \neq \operatorname{sur-}$ rounded $\left(\sigma_{j}, \sigma_{i}\right)$. It can be seen that $\operatorname{surrounded}\left(\sigma_{i}, \sigma_{j}\right) \Rightarrow j \operatorname{joint}\left(\sigma_{i}, \sigma_{j}\right)$. Definition 5:

$$
\begin{array}{r}
\text { unrelated }\left(\sigma_{i}, \sigma_{j}\right) \Leftrightarrow \neg\left(\operatorname{surrounded}\left(\sigma_{i}, \sigma_{j}\right) \vee\right. \\
\left.\operatorname{surrounded}\left(\sigma_{j}, \sigma_{i}\right) \vee j \operatorname{joint}\left(\sigma_{i}, \sigma_{j}\right)\right)
\end{array}
$$

\subsection{Graph Representation of $\Sigma$}

Definition 6: The graph representation of $\Sigma$ is a graph $G=(V, A)$, where $V=\left\{v_{1}, \cdots, v_{|\Sigma|}\right\}$ is a set of vertices corresponding to $\left\{\sigma_{1}, \cdots, \sigma_{|\Sigma|}\right\}$ and $A=\left\{\cdots, a_{i j}, \cdots\right\}$ a set of arcs such that $a_{i j}$ is an undirected arc 
between $v_{i}$ and $v_{j}$ when joint $\left(\sigma_{i}, \sigma_{j}\right)$ is true but a directed arc from $v_{j}$ to $v_{i}$ when surrounded $\left(\sigma_{i}, \sigma_{j}\right)$ is true and vice versa.

Theoretically, as long as $|\Sigma|$ is finite, the number of the possible structures of $\Sigma$ will be finite. However, experimental results have shown that a graph representation has the following shortcomings ${ }^{6}$ :

- A graph representation is not a hierarchical structure in most cases.

- The correspondence between isostructural $\Sigma$ 's and their graph representations may not be one-to-one.

- The number of the possible structures and also the corresponding graph representations overwhemingly increase as $|\Sigma|$ increases.

This is because a graph representation is a completely machine-oriented structure representation.

\subsection{Partition of $\Sigma$}

Let $\Psi_{\Sigma}=\left\{\Sigma_{m}|1 \leq m \leq| \Psi_{\Sigma} \mid\right\}$ be a set which consists of the subsets of $\Sigma$ such that in the case $\left|\Sigma_{m}\right|>1, \Sigma_{m}$ satisfies:

$$
\forall \sigma_{p} \exists \sigma_{q}\left(M\left(\sigma_{p}, \Sigma_{m}\right) \wedge M\left(\sigma_{q}, \Sigma_{m}\right) \Rightarrow j \operatorname{joint}\left(\sigma_{p}, \sigma_{q}\right)\right)
$$

Definition 7: $\psi_{\Sigma} \subseteq \Psi_{\Sigma}$ is a partition of $\Sigma$ iff $\forall \Sigma_{p}, \forall \Sigma_{q} \in \psi_{\Sigma} \Sigma_{p} \cap \Sigma_{q}=\phi$ and $\cup_{i=1}^{\left|\psi_{\Sigma}\right|} \Sigma_{i}=\Sigma$.

Obviously, $\left\{\left\{\sigma_{i}\right\}|1 \leq i \leq| \Sigma \mid\right\}$ is the case that $\forall \Sigma_{m} \in \psi_{\Sigma}\left|\Sigma_{m}\right|=1$ and hence a candidate for $\psi_{\Sigma} . \psi_{\Sigma}$ is non-deterministic unless $|\Sigma|=1$.

Let $\Delta_{m}$ be a set of pixels corresponding to $\Sigma_{m} \in \Psi_{\Sigma}$ such that $\Delta_{m}=\cup \sum_{i=1}^{\left|\Sigma_{m}\right|} \sigma_{m_{i}}$. The difference between $\Delta_{m}$ and $\Sigma_{m}$ is the same as that between $\Delta$ and $\Sigma$, i.e., the elements of $\Delta_{m}$ and $\Delta$ are pixels but the elements of $\Sigma_{m}$ and $\Sigma$ are GCC's. Def. 2 is generalized as follows:

Definition 8: The border $\Delta_{m}^{\prime}$ of $\Delta_{m}$ is a subset of $\Delta_{m}$ such that $\Delta_{m}^{\prime}=$ $\left\{p \mid \exists q \in \Delta^{\prime} \exists \pi \Delta_{m} \cap \pi=\{p\}\right\}$, where $\pi$ is a path between $p$ and $q$ and $\{p\}$ denotes a set with a single pixel $p$.

The correspondence between $\Sigma_{m}$ and $\Delta_{m}^{\prime}$ is not always one-to-one because we may have $\exists \sigma_{p} \in \Sigma_{m}$ but $\sigma_{p} \cap \Delta_{m}^{\prime}=\phi$. The following constraint is thus defined to uniquely determine $\Sigma_{m} \in \Psi_{\Sigma}$ for a given $\Delta_{m}^{\prime}$.

Constraint 1: $\Sigma_{m}=\left\{\sigma_{i} \mid \sigma_{i} \cap \Delta_{m}^{\prime} \neq \phi\right\}$.

Now we can generalize Def. 3 as follows:

Definition 9: $\forall \Sigma_{m} \in \Psi_{\Sigma}$ satisfying Con.1 and $\forall \Sigma_{n} \in \Psi_{\Sigma}$, surrounded $\left(\Sigma_{m}, \Sigma_{n}\right)$ is true iff $\forall \sigma_{q} \in \Sigma_{n} \sigma_{q} \cap B_{\Delta_{m}^{\prime}} \neq \phi$ and $\Delta_{n} \supseteq B_{\Delta_{m}^{\prime}}$, where $B_{\Delta_{m}^{\prime}}=\left\{p \mid N_{b}\left(p, \Delta_{m}^{\prime}\right)\right\}$. 
The following are the human-oriented constraints, which actually determine a procedure for the generation of a unique partition $\psi_{\Sigma}^{U}$.

Constraint 2: If $\Sigma_{1}=\left\{\sigma_{i} \mid \sigma_{i} \cap \Delta^{\prime} \neq \phi\right\}$ then $\Sigma_{1} \in \psi_{\Sigma}^{U}$.

Constraint 3: $\forall \Sigma_{m} \in \psi_{\Sigma}^{U}$ with $m \geq 1$ and $\forall \Sigma_{n} \in \Psi_{\Sigma}$ with $n>m$, if $\exists S_{\Sigma_{m}} \subseteq \Sigma_{m}$ and surrounded $\left(\Sigma_{n}, S_{\Sigma_{m}}\right)$ is true then $\Sigma_{n} \in \psi_{\Sigma}^{U}$.

\subsection{A Tree Structure Representation of $\Sigma$}

Definition 10: $T=\left(V_{T}, A_{T}\right)$, where $V_{T}=\left\{V_{1}, \cdots, V_{\left|\psi_{\Sigma}^{U}\right|}\right\}$ is a set of vertices corresponding to $\left\{\Sigma_{1}, \cdots, \Sigma_{\left|\psi_{\Sigma}^{U}\right|}\right\}$ and $A_{T}=\left\{\cdots, A_{i j}, \cdots\right\}$ a set of directed arcs such that $A_{i j}$ is directed from $V_{j}$ to $V_{i}$ in the case that surrounded $\left(\Sigma_{i}, S_{\Sigma_{j}}\right)$ with $S_{\Sigma_{j}} \subseteq \Sigma_{j}$ is true and vice versa.

The difference between $T=\left(V_{T}, A_{T}\right)$ and $G=(V, A)$ is as follows.

- $V=\left\{v_{1}, \cdots, v_{i}, \cdots, v_{|\Sigma|}\right\}$, where $v_{i}$ corresponds to $\sigma_{i}$ and $|\Sigma|$ is the total number of GCC's in $\Sigma$, but $V_{T}=\left\{V_{1}, \cdots, V_{i}, \cdots, V_{\mid \psi_{\Sigma}^{W}}\right\}$, where $V_{i}=\left\{X_{i}, E_{i}\right\}$ is a graph which corresponds to $\Sigma_{i}$ and $\left|\psi_{\Sigma}^{U}\right|$ is the total number of graphs. Further, $X_{i}=\left\{x_{i 1}, \cdots, x_{i\left|\Sigma_{i}\right|}\right\}$ is a set of vertices representing $\left\{\sigma_{i 1}, \cdots, \sigma_{i\left|\Sigma_{i}\right|}\right\}$ and $E_{i}=\left\{\cdots, e_{(i p)(i q)}, \cdots\right\}$ a set of undirected arcs such that $e_{(i p)(i q)}$ linking $x_{i p}$ and $x_{i q}$ corresponds to the case that joint $\left(\sigma_{i p}, \sigma_{i q}\right)$ is true.

- $A=\left\{\cdots, a_{i j}, \cdots\right\}$, where $a_{i j}$ can be either directed or undirected depending on whether joint $\left(\sigma_{i}, \sigma_{j}\right)$ or surrounded $\left(\sigma_{i}, \sigma_{j}\right)$ (or sur$\left.\operatorname{rounded}\left(\sigma_{j}, \sigma_{i}\right)\right)$ is true. On the other hand, $A_{T}=\left\{\cdots, A_{i j}, \cdots\right\}$, where $A_{i j}$ can only be a directed arc because either surrounded $\left(\Sigma_{i}, S_{\Sigma_{j}}\right)$ with $S_{\Sigma_{j}} \subseteq \Sigma_{j}$ or surrounded $\left(\Sigma_{j}, S_{\Sigma_{i}}\right)$ with $S_{\Sigma_{i}} \subseteq \Sigma_{i}$ must be true. In the case that surrounded $\left(\Sigma_{j}, \Sigma_{i}\right)$ is true, $A_{i j}$ is directed from $V_{i}$ to $V_{j}$. In the case that surrounded $\left(\Sigma_{j}, S_{\Sigma_{i}}\right)$ with $S_{\Sigma_{i}} \subset \Sigma_{i}$ is true, however, $A_{i j}$ is directed from a subgraph but not the whole of $V_{i}$ to $V_{j}$, where the subgraph corresponds to $S_{\Sigma_{i}}$.

\section{Content-based indexing and retrieval}

The transformation from $\Delta$ to $\Sigma$ is a pixel grouping process. We simplify the pixel grouping problem by using a document image database created from Ref.7. Fig.1 shows a computer-generated $\Sigma$ and its corresponding structure representation.

Fig.2(a) shows a key image whose structure representation is used as a key for a retrieval experiment. Fig.2(b) shows the first candidate retrieved. Fig.2(c) shows the second candidate. The retrieval process is 
basically a matching processing between the structure representations. The results shown in Fig.2(b) and Fig.2(c) are obtained by using the following to parameters.

- $\left|F_{\Sigma_{j}}\right|, F_{\Sigma_{j}}=\left\{\Sigma_{i} \mid\right.$ surrounded $\left.\left(\Sigma_{i}, S_{\Sigma_{j}}\right)\right\}$, i.e., the number of elements which are surrounded by the same element.

- $\left|\Sigma_{i}\right|$, the number of GCC's in $\Sigma_{i}$, i.e., a particular element.

\section{Conclusion}

A new structure representation of an image has been presented. Except for the human-oriented constraints for uniquely generating GCC groups, the overall process for the generation of the structure representation is a data-driven process. Since the structure representation is a hierarchy, a coarse-to-fine pattern matching is possible.
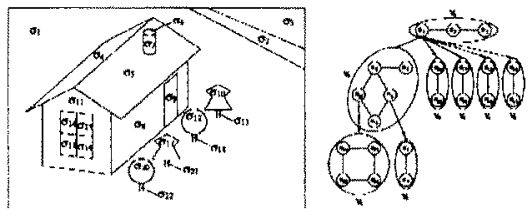

Figure 1: An example image and its structure representation.

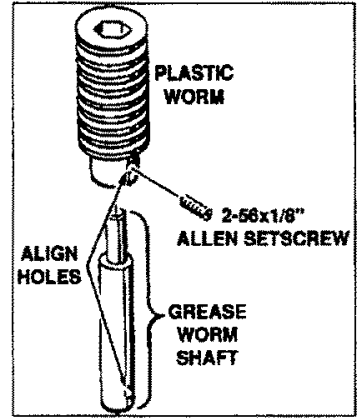

(a)

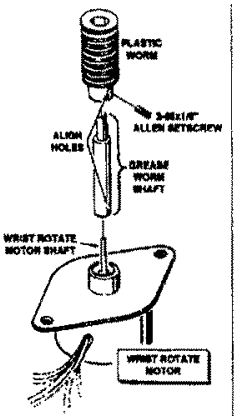

(b)

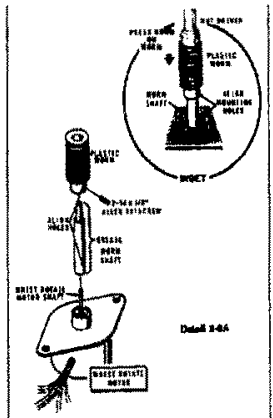

(c)

Figure 2: (a) a key image for an image retrieval experiment; (b) the first candidate retrieved; $(c)$ the second candidate retrieved. 


\section{References}

1. K. S. Fu. Syntactic Pattern Recognition, Applications. Springer-Verlag, 1977.

2. D. Marr. Vision. Freeman and Company, New York, 1982.

3. H.J. Zhang, et al. "Video parsing, retrieval, and browsing: an integrated and content-based solution," Proc. ACM Multimedia'95, 1995.

4. A. K. Jain. Fundamentals of Digital Image Processing. Prentice Hall, Inc., 1989.

5. A. Rosenfeld and A.C. Kak. Digital Picture Processing. 2nd Edition, Vol. 2, Chapter 11, Academic Press, Inc., 1982.

6. S. He and N. Abe. "A Practical Structural Representation of a Segmented Image," IEICE Trans. on Information and Systems, vol. E79-D, no. 6, pp. 524-531, June. 1996.

7. HERO ROBOT Arm Accessary Manual, Health Company, Michigan, 1982. 